\title{
Optimization of Gigabit Passive Optical Network (GPON) Devices Replacement Interval
}

\author{
Muhammad Mahrus Syamsurrijal, Abdullah Shahab \\ Department of Technology Management, Institut Teknologi Sepuluh Nopember, Indonesia \\ e-mail:m.m.syamsurrijal@gmail.com
}

\begin{abstract}
Entering the industrial era that is increasingly advanced and growing, reliable internet connection becomes a very important foundation for every business. Internet connection failure or downtime will be the obstacle and causes much loss both for businessman and for internet provider company. This research aims to find a solution on how to minimize internet connection failure. One of the various methods that can be used to overcome the problem is called preventive maintenance. This research is chosen to determine how far the role of preventive maintenance in reducing the risk of connection failure due to device failure. Several tools that used in this research, such as statistical distribution to determine the pattern of device failure, and mathematical formula to calculate the estimated duration of the downtime occurs based on the time of preventive maintenance was applied. From that calculation, it can be concluded the optimal time to apply preventive maintenance to minimize downtime duration. The results reveal that a decreasing downtime value happens when preventive maintenance is applied at the optimal age of the device. EI8E 8FE Electrical Interface Card kit was reduced by $8.01 \%$ with an optimal age of 21.82 days. GTGH - 16 Port GPON Line Card devices were reduced by $5.46 \%$ with an optimal age of 16.69 days. EMTPDP-DC Power Board devices are reduced by $0.1 \%$ with an optimal age of 53 days. The GPON MSVG - Control \& Switching Card device is reduced by $4.38 \%$ with an optimal age of 32.28 days. MTPD - Power Supply equipment is reduced by $1.2 \%$ with an optimal age of 92.35 days. The ETC8B - 8E1 / T1 CES Card device was reduced by $0.84 \%$ with an optimal age of 41.23 days.
\end{abstract}

Keywords-Data Processin, Downtime, Internet, Preventive Maintenance.

\section{INTRODUCTION}

$\mathrm{R}$ ELIABILITY is one of the eight dimensions that determines quality in both industries that produce goods and service industries [1]. In the internet service industry, one of the metrics used to measure reliability is how long the internet connection service can remain connected (uptime). This measure is represented as a percent (\%) per unit time. The greater the uptime value of an internet service, the better the reliability. In other words, the smaller the value of downtime (connection failure), the better the reliability. The reliability factor that will be discussed in this research focus on how to minimize downtime due to device failure. This research takes Gigabit Passive Optical Network (GPON) devices as a research object because GPON internet network consists of various devices and modules that are prone to damage. So that if there is damage to one of the devices, then the internet service will experience interference that causes the value of downtime to increase. Besides, GPON technology is a technology that is still commonly used by internet service providers in Indonesia.

Preventive maintenance is used as the method to achieve minimum downtime value of GPON internet network. Several tools that used in this research, are statistical distribution and mathematical formula. Statistical distribution is used for data processing so that the device failure data can be converted into data distribution all in with its parameters. This statistical distribution and its parameters will be used in mathematical formula to calculate the estimated duration of the downtime occurs based on the time of preventive maintenance was applied.

GPON is a PON-based optical fiber local access network technology standardized by ITU-T [2]. The main components of the GPON technology are as follows:

1) Network Management System (NMS)

2) NMS is software to control and configure GPON devices. NMS can configure both on the OLT and ONT sides. In addition, NMS can manage services running on GPON technology such as internet, telephone and IPTV.

3) Optical Line Terminal (OLT)

4) OLT is an active GPON device on the server-side to convert electrical signals into optical signals. There are several modules on the OLT that support telecommunications facilities, including control modules, customer modules, transport modules, and power supply modules.

5) Optical Network Terminal (ONT)

6) ONT is an active GPON device on the customer-side to connect the customer's device with the GPON network.

7) Optical Distribution Cabinet (ODC)

8) ODC is a passive device as a branching point from a large capacity cable (feeder) into several cables with a smaller capacity (distribution cable).

9) Optical Distribution Point (ODP)

ODP is a passive device that functions as a branching point from the distribution cable into several cables that connect with GPON active devices on the customer-side.

10) Splitter

Splitter is a passive device to divide the optical signal into several lines with smaller capacity.

Table 1 shows the GPON devices which failed frequently based on maintenance unit, and how many customers will be affected.

\section{METHOD}

Weibull ++ software is used for reliability analysis, while Wolfram Mathematica software is used for devices 
The $1^{\text {st }}$ International Conference on Business and Engineering Management (IConBEM)

February $1^{\text {st }} 2020$, Institut Teknologi Sepuluh Nopember, Surabaya, Indonesia

Table 1.

GPON Devices with Number of Failure and Number of Customer Affected

\begin{tabular}{lccc}
\hline \hline \multirow{2}{*}{ Devices } & $\begin{array}{l}\text { Frequency of } \\
\text { Failure (Times) }\end{array}$ & $\begin{array}{l}\text { Customer Potentially Affected by } \\
\text { Each Failure (Customers) }\end{array}$ & $\begin{array}{l}\text { Sum of Customer Potentially } \\
\text { Affected (Customer) }\end{array}$ \\
\hline EMTPDP - DC Power Board & 106 & 128 & 13568 \\
MSVG - Control \& Switching Card GPON & 75 & 128 & 9600 \\
MTPD - Power Supply & 46 & 128 & 5888 \\
GTGH - 16 Port GPON Line Card & 112 & 16 & 1792 \\
EI8E - 8FE Electrical Interface Card & 192 & 8 & 1536 \\
ETC8B - 8E1/T1 CES Card & 40 & 8 & 320 \\
ONT HWI HG8245A & 218 & 1 & 218 \\
ONT HUAWEI SMARTAX MA5694 & 185 & 1 & 185 \\
ONT ZXA10 F829 - (4GE-2G-DC) & 178 & 1 & 178 \\
ONT ZXA10 F829 2DC48 & 94 & 1 & 94 \\
ONT ZXA10 F829 & 84 & 1 & 84 \\
ONT ZXA10 F821 - MULTI TENANT & 76 & 1 & 76 \\
ONT ZXA10F820 & 66 & 1 & 66 \\
ONT ALU G-240W-A & 31 & 1 & 31 \\
ONT ZXA10 F821 DC & 20 & 1 & 20 \\
\hline \hline
\end{tabular}

Table 2.

Weibull Probability Density Function

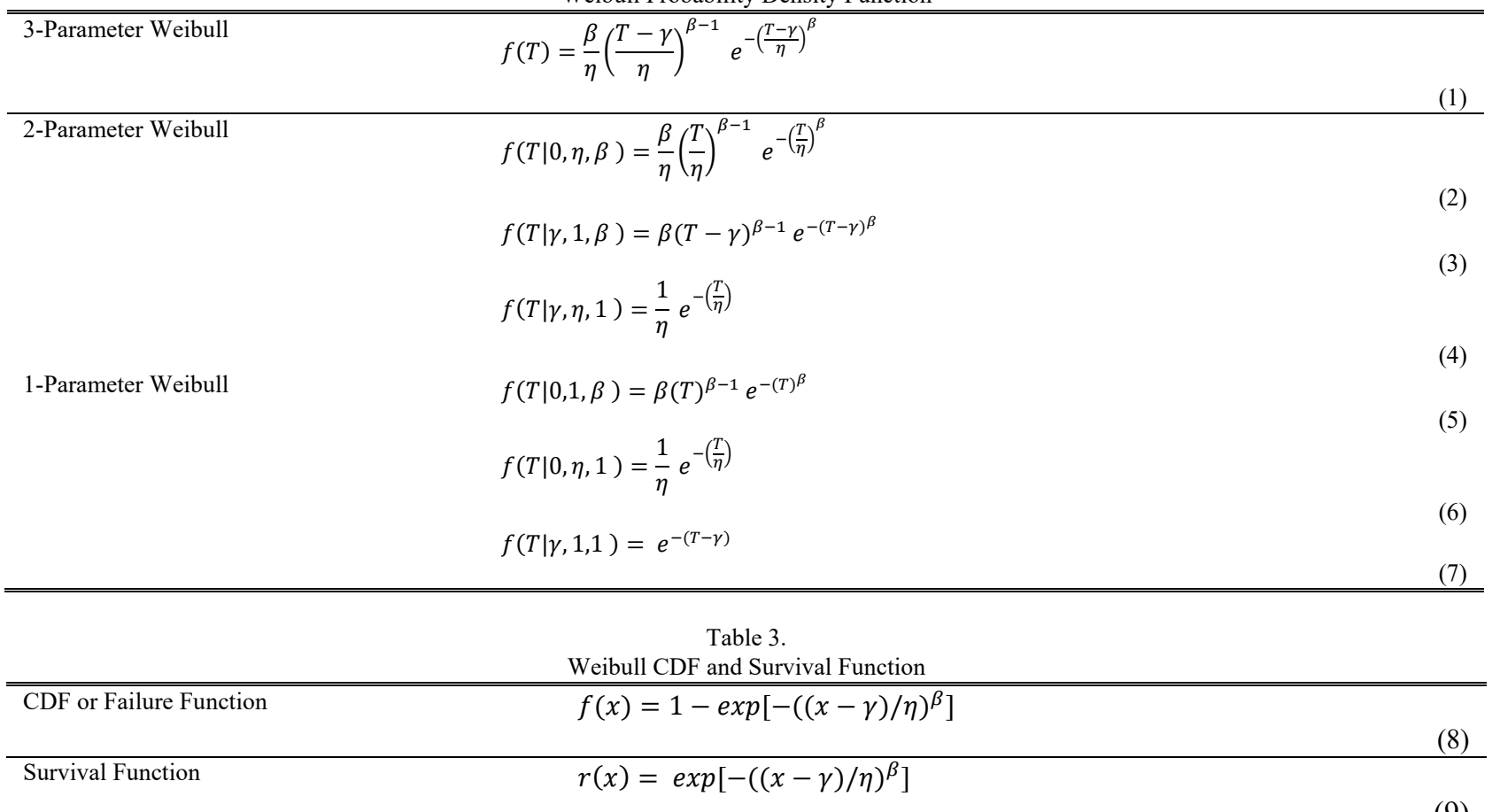

replacement optimization. The workflow of the research was as follow:

1) Collecting device failure data

2) Analyze critical devices with Pareto chart based on the number of customers potentially affected

3) Convert device failure data into time between failures

4) Determine the distribution of time between failures using Weibull++ software

5) Calculate downtime value for each preventive maintenance and corrective maintenance

6) Determine the optimum interval and downtime value for device replacement

A. Pareto Principle

Pareto principle or also known as the $80 / 20$ rule states that for many cases, $80 \%$ of the wastes or losses are due to $20 \%$ of the causes. Pareto principle can help find and sort issues from the urgent issues to be solved to the minor issues to be solved.
The application of Pareto principle is a Pareto chart, which is a bar graph to visually shows which situations are more significant [3]. In this research, Pareto chart was used to analyze which of these GPON devices are critical. While the criteria that used to rank in this Pareto chart is the number of customers potentially affected by device failure. The device that potentially affects the bigger number of customers should have a higher rank. On the opposite, the device that potentially affects the smaller number of customers should have a lower rank. Figure 1 shows Pareto chart of customers potentially affected by device failure. This research takes the top six devices to be the research object, which are:
1) EMTPDP - DC Power Board
2) MSVG - Control \& Switching Card GPON
3) MTPD - Power Supply
4) GTGH - 16 Port GPON Line Card
5) EI8E - 8FE Electrical Interface Card 
Table 4.

Truncated Weibull Distribution Mean Formula

Doubly-truncated two-parameter Weibull distribution

$$
\begin{gathered}
E\left(X_{l, r}\right)=\frac{\beta}{e^{-\left(\frac{l}{\beta}\right)^{\alpha}}-e^{-\left(\frac{r}{\beta}\right)^{\alpha}}\left[\gamma\left(\frac{1}{\alpha}+1,\left(\frac{r}{\beta}\right)^{\alpha}\right)-\gamma\left(\frac{1}{\alpha}+1,\left(\frac{l}{\beta}\right)^{\alpha}\right)\right]} \\
\text { Left-truncated two-parameter Weibull distribution } \\
E\left(X_{l}\right)=\frac{\beta}{e^{-\left(\frac{l}{\beta}\right)^{\alpha}}\left[\Gamma\left(\frac{1}{\alpha}+1\right)-\gamma\left(\frac{1}{\alpha}+1,\left(\frac{l}{\beta}\right)^{\alpha}\right)\right]}
\end{gathered}
$$

Right-truncated two-parameter Weibull distribution

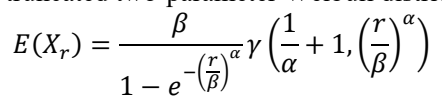

Doubly-truncated three-parameter Weibull distribution

$$
E\left(Y_{l, r}\right)=E\left(X_{l-\theta, r-\theta}\right)+\theta
$$

Left-truncated three-parameter Weibull distribution

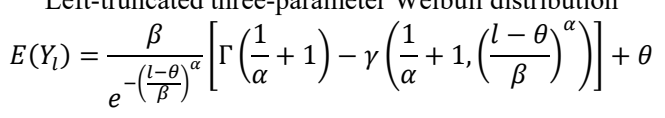

Right-truncated three-parameter Weibull distribution

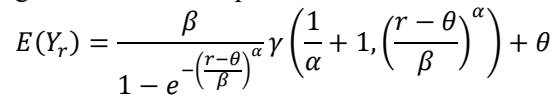

\section{6) ETC8B - 8E1/T1 CES Card \\ B. Weibull Distribution}

Weibull distribution is a distribution that has an important role, especially for reliability analysis. Weibull distribution is often used as an approach to determine the characteristics of the failure function because changes in value will result in this distribution having certain properties or equivalent to certain distributions. This distribution is a multipurpose distribution that can take characteristics from other types of distribution, based on the value of the shape parameter [4]. Figure 2 shows the example of Weibull distribution graph. Mathematically, the distribution is defined by its Probability Density Function (PDF). PDF that generally used to define Weibull distribution is the three-parameters formula. Table 2 shows three kinds of Weibull PDF.

Another property of a distribution is Cumulative Density Function (CDF). CDF is the probability that a variable in the distribution will take a value less than or equal to a point where the distribution is evaluated. Table 3 shows the formula for Weibull $\mathrm{CDF}$ and survival function. 1

where $f(T) \geq 0, T \geq 0$ or $\gamma, \beta \geq 0, \eta \geq 0,-\infty<\gamma<\infty$ and,

1. $\beta$ is shape parameter, or also known as Weibull Slope

2. $\eta$ is scale parameter

3. $\gamma$ is location parameter

For some cases are often confronted to censored data sample in these fields, the truncated Weibull distribution functions can be great interest. A truncated distribution is defined on an interval that is only a sub-interval of the complete distribution [5]. Figure 3 shows both Weibull distribution and truncated Weibull distribution. The properties of truncated Weibull distribution are not the same as the complete Weibull distribution. This research is using only the mean of truncated
Weibull distribution to calculate optimal downtime. Table 4 is the mean formula for truncated Weibull distribution.

Where the incomplete gamma function,

$$
\gamma(z, u)=\int_{0}^{u} x^{z-1} e^{-x} d x
$$

and,

$$
\lim _{u \rightarrow+\infty} \gamma(z, u)=\Gamma(z)
$$

C. Age-Replacement Model

Age-replacement model is a replacement model where the time interval for device or component replacement is done by considering the service life of the component, to avoid replacing newly installed equipment will be replaced in a relatively short time [6]. Usually, replacement of the device or components at certain intervals or ages is done to minimizing costs, both replacement costs and operational costs. However, there are several cases when device or component replacement at certain intervals or ages is more aimed at minimizing the downtime that occurs [7]. Figure 4 compares the time interval for replacing a device before it is damaged and when the device is damaged. The earlier the time interval for replacing devices or parts, the greater the preventive costs of replacing the device or parts. Whereas if the device fails before the preventive replacements, then the losses incurred due to damage to the device become greater. So, a balance is required between the amount spent on the preventive replacements and their resulting benefits, that is, reduced failure replacements. The conflicting downtime consequences and their resolution by identifying the total cost curve are illustrated in Figure 5.

The total downtime per unit time for preventive replacements once the item becomes of age $t p$ is:

$$
D\left(t_{p}\right)=\frac{\text { Total expected downtime } / \text { cycle }}{\text { Expected cycle length }}
$$

where, 
The $1^{\text {st }}$ International Conference on Business and Engineering Management (IConBEM)

February $1^{\text {st }} 2020$, Institut Teknologi Sepuluh Nopember, Surabaya, Indonesia

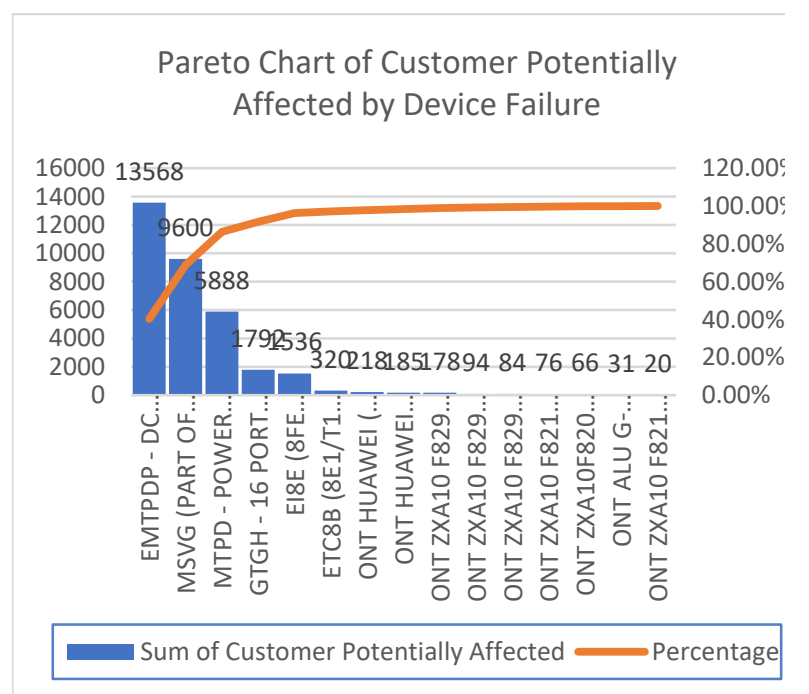

Figure 1. Pareto Chart of Customer Potentially Affected by Device Failure.

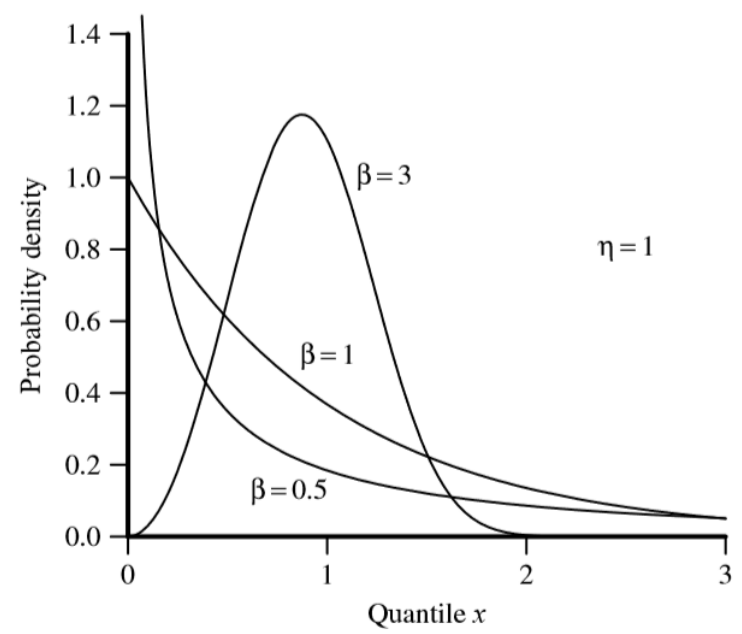

Figure 2. Weibull Distribution Graph.

1) Total expected downtime/cycle
a. $=$ downtime due to a preventive cycle $\times$ probability of a preventive cycle + downtime due to a failure cycle $\times$ probability of a failure cycle

b. $=T_{p} R\left(T_{p}\right)+T_{f}\left[1-R\left(T_{p}\right)\right]$

2) Expected cycle length

a. = length of a preventive cycle $\times$ probability of a preventive cycle + expected length of a failure cycle $\times$ probability of a failure cycle

b. $=\left(t_{p}+T_{p}\right) \times R\left(T_{p}\right)+\left[M\left(t_{p}\right)+T_{f}\right] \times\left[1-R\left(T_{p}\right)\right]$

3) $M\left(t_{p}\right)=$ Mean time to failure if a preventive replacement occurs at time $t_{p}$

Thus, the model of determining the age of component replacement with the aim of minimizing downtime can be written as formulated by Jardine \& Tsang [7] below:

$$
D\left(t_{p}\right)=\frac{T_{p} R\left(T_{p}\right)+T_{f}\left[1-R\left(T_{p}\right)\right]}{\left(t_{p}+T_{p}\right) \times R\left(T_{p}\right)+\left[M\left(t_{p}\right)+T_{f}\right] \times\left[1-R\left(T_{p}\right)\right]}
$$

where, $\begin{array}{ll}t_{p} & =\text { The optimal age at which preventive } \\ & \text { replacements should occur }\end{array}$

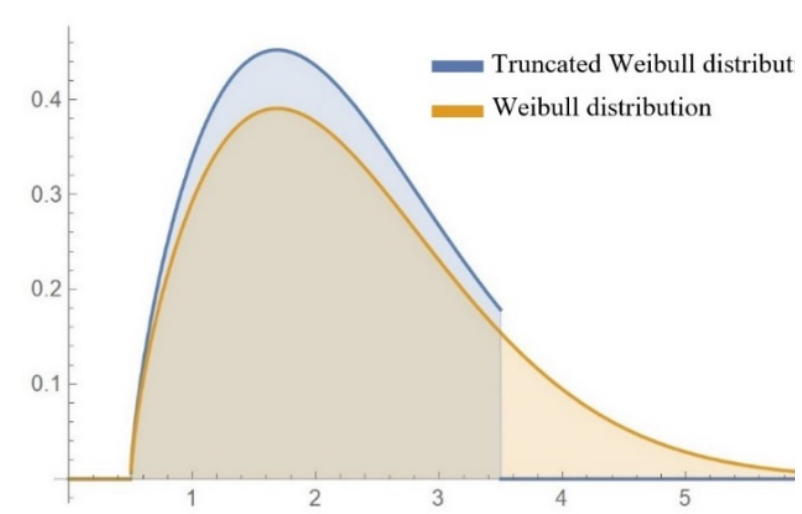

Figure 3. Truncated Weibull Distribution.

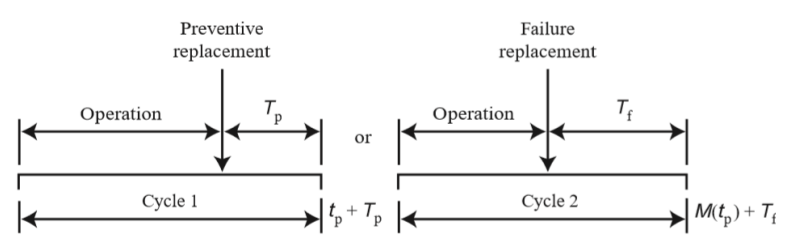

Figure 4. Age-based Replacement Cycle.

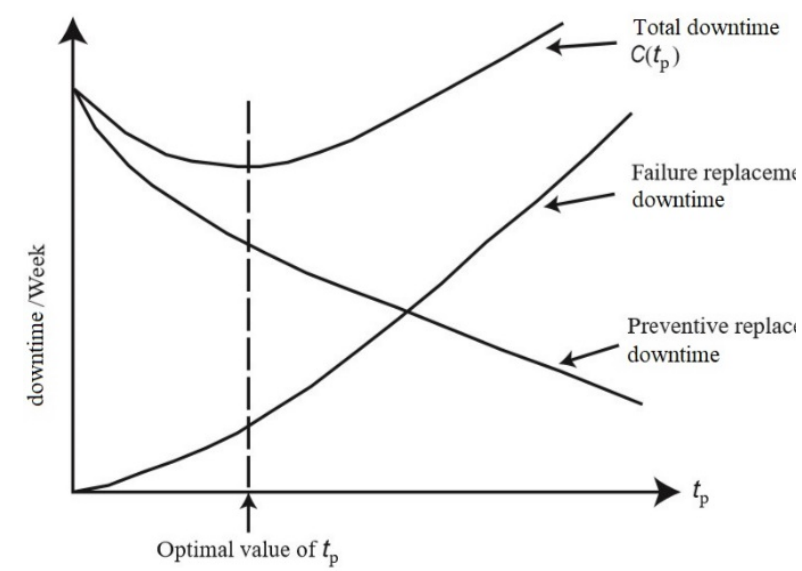

Figure 5. Optimal Replacement Time.

$T_{p}=$ Downtime due to a preventive cycle

$T_{f}=$ Downtime due to a failure cycle

$R\left(t_{p}\right)=$ Probability of a preventive cycle

$M\left(t_{p}\right) \quad=\quad$ Mean time to failure if a preventive replacement occurs at time $t_{p}$.

The mean of shaded portion of a truncated Weibull distribution on Figure 3, because there is the only possible region for failure before preventive replacement

\section{RESULTS AND DISCUSSION}

The data used as the research object is device failure data of the chosen device, obtained from the company database which then be converted into Time Between Failures (TBF). As an example, the TBF list for GTGH - 16 Port GPON Line Card is shown in Table 5. Based on the TBF data for each of the GPON devices, the result of distribution test in Weibull++ software to determine the most appropriate distribution function is shown in Table 6. While the parameters for each GPON devices distribution is shown in Table 7. 
The $1^{\text {st }}$ International Conference on Business and Engineering Management (IConBEM)

February $1^{\text {st }} 2020$, Institut Teknologi Sepuluh Nopember, Surabaya, Indonesia

Table 5.

Time Between Failures (TBF) data for GTGH-16 Port GPON Line Card

\begin{tabular}{ccccccccccc}
\hline \hline & \multicolumn{10}{c}{ Time Between Failure GTGH - 16 Port GPON Line Card (Hari) } \\
\hline 22 & 18 & 5 & 7 & 1 & 1 & 1 & 14 & 11 & 106 & 8 \\
31 & 1 & 15 & 6 & 1 & 3 & 13 & 13 & 7 & 2 & 47 \\
13 & 64 & 2 & 3 & 1 & 8 & 15 & 20 & 8 & 40 & 146 \\
28 & 28 & 5 & 11 & 3 & 3 & 10 & 13 & 6 & 9 & 56 \\
20 & 7 & 6 & 14 & 11 & 3 & 7 & 14 & 1 & 51 & 7 \\
2 & 11 & 11 & 9 & 5 & 17 & 1 & 1 & 201 & 24 & 202 \\
11 & 21 & 3 & 5 & 14 & 20 & 13 & 20 & 33 & 73 & 14 \\
16 & 3 & 9 & 16 & 7 & 1 & 3 & 4 & 42 & 97 & 21 \\
6 & 6 & 1 & 22 & 9 & 8 & 28 & 27 & 10 & 14 & \\
9 & 28 & 6 & 25 & 20 & 13 & 7 & 14 & 19 & 25 & \\
\hline \hline
\end{tabular}

Table 6.

Distribution Test Results

\begin{tabular}{|c|c|c|c|c|c|c|c|}
\hline \multirow{2}{*}{ Devices } & \multicolumn{6}{|c|}{ Rank } & \multirow{2}{*}{ Distribution } \\
\hline & Exponential 2 & Exponential 1 & Normal & Lognormal & Weibull 2 & Weibull 3 & \\
\hline EI8E - 8FE Electrical Interface Card & 3 & 2 & 6 & 5 & 1 & 4 & Weibull 2 \\
\hline GTGH - 16 Port GPON Line Card & 5 & 4 & 6 & 2 & 3 & 1 & Weibull 3 \\
\hline EMTPDP - DC Power Board & 4 & 6 & 5 & 3 & 1 & 2 & Weibull 2 \\
\hline MSVG - Control \& Switching Card GPON & 4 & 3 & 6 & 5 & 1 & 2 & Weibull 2 \\
\hline MTPD - Power Supply & 3 & 4 & 6 & 5 & 1 & 2 & Weibull 2 \\
\hline ETC8B - 8E1/T1 CES Card & 3 & 4 & 6 & 2 & 5 & 1 & Weibull 3 \\
\hline
\end{tabular}

Table 7.

Parameters of GPON Devices Distribution

\begin{tabular}{llll}
\hline \hline \multicolumn{1}{c}{ Devices } & \multirow{2}{*}{ Distribution } & \multicolumn{2}{c}{ Parameter } \\
\cline { 2 - 4 } & & Form, $\beta$ & Scale, $\eta$ \\
\hline EI8E - 8FE Electrical Interface Card & Weibull 2 & 1.0626 & 67.0009 \\
GTGH - 16 Port GPON Line Card & Weibull 3 & 0.8659 & 16.6375 \\
EMTPDP - DC Power Board & Weibull 2 & 0.9143 & 71.0022 \\
MSVG - Control \& Switching Card GPON & Weibull 2 & 1.1124 & 76.3834 \\
MTPD - Power Supply & Weibull 2 & 0.925 & - \\
ETC8B - 8E1/T1 CES Card & Weibull 3 & 0.9939 & - \\
\hline \hline
\end{tabular}

Table 8.

Optimum Replacement Time and Minimum Downtime

\begin{tabular}{lccc}
\hline \hline \multicolumn{1}{c}{ Devices } & Distribusi & Downtime (days) & Interval (days) \\
\hline EI8E-8FE Electrical Interface Card & Weibull 2 & 0.005630 & 21.815869 \\
GTGH-16 Port GPON Line Card & Weibull 3 & 0.026387 & 16.692354 \\
EMTPDP-DC Power Board & Weibull 2 & 0.006150 & 53.057394 \\
MSVG-Control \& Switching Card GPON & Weibull 2 & 0.005263 & 32.276935 \\
MTPD-Power Supply & Weibull 2 & 0.003262 & 92.347698 \\
ETC8B-8E1/T1 CES Card & Weibull 3 & 0.003305 & 41.234831 \\
\hline \hline
\end{tabular}

For the GTGH - 16 Port GPON Line Card, which has Weibull 3 distribution, the appropriate downtime estimation could be calculated with Equation 16, with Equation 8, 9 and 15 as complementary. Using those equation, downtime data input (which consists of preventive replacement downtime and corrective replacement downtime), and the device's TBF distribution parameters, the optimum replacement time which will result in minimum average downtime could be calculated. Using those equations and Wolfram Mathematica software, the optimum replacement time and cost graph for GTGH - 16 Port GPON Line Card was acquired. Figure 6 shows the change in GTGH - 16 Port GPON Line Card downtime as a function of time. The $\mathrm{Y}$-axis shows the cost in Days or minute/day, and the $\mathrm{X}$-axis shows time in days. The orange curve is the corrective maintenance downtime, while the blue curve is the preventive maintenance downtime, and the light green curve is the total downtime which is a sum of corrective and preventive downtime. Figure 6 shows that the longer the component replacement time, the lower the preventive maintenance downtime would be. On the other hand, the corrective maintenance downtime would increase if the component replacement interval is longer. The minimum total downtime was found at the interval of 16.692354 days, with the average downtime of 0.026387 days or equal to 38 minutes per day.

Using the same workflow, the optimum time and minimum cost for GPON devices maintenance can be calculated. Table 8 shows the results of the calculations for each device.

The optimum replacement time and minimum downtime value is affected by various factors, such as the amount of personnel required to do the maintenance, the time required to do the replacement, equipment malfunction time, the quality of the device, and the data distribution parameters itself. But it is better to do the replacements around the optimum time to reduce the downtime value. For devices with similar optimum replacement interval, preventive maintenance can be done at the same time. As an example, MSVG - Control \& Switching Card GPON and EI8E - 8FE Electrical Interface Card has the optimum interval of 32.276935 days and 21.815869 days respectively. 
The $1^{\text {st }}$ International Conference on Business and Engineering Management (IConBEM)

February $1^{\text {st }} 2020$, Institut Teknologi Sepuluh Nopember, Surabaya, Indonesia

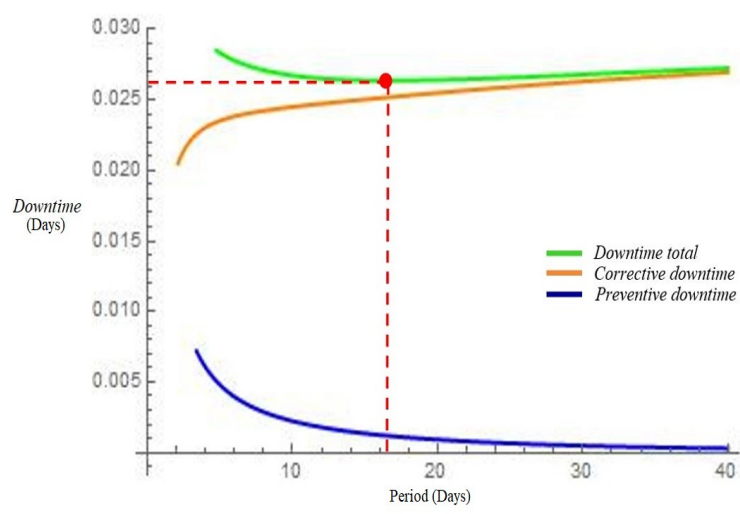

Figure 6. GTGH - 16 Port GPON Line Card Replacement Downtime Plot.

\section{CONCLUSIONS}

Based on the research, there are some conclusions that can be made:

A. Of all GPON devices failure data, 4 devices have Weibull 2 distribution, and 2 devices has Weibull 3 distribution.

B. The optimum replacement time for GPON devices are as follows:

1) EMTPDP - DC Power Board should be replaced after 53.057394 days.

2) MSVG - Control \& Switching Card GPON should be replaced after 32.276935 days.

3) MTPD - Power Supply should be replaced after 92.347698 days.

4) GTGH - 16 Port GPON Line Card should be replaced after 16.692354 days.

5) EI8E - 8FE Electrical Interface Card should be replaced after 21.815869 days

6) ETC8B - 8E1/T1 CES Card should be replaced after 41.234831 days.

C. The optimum downtime value for GPON devices are as follows:

1) EMTPDP - DC Power Board has optimum downtime value for 0.0062 days or equal to 9 minutes.
2) MSVG - Control \& Switching Card GPON has optimum downtime value for 0.0053 days or equal to 7 minutes.

3) MTPD - Power Supply has optimum downtime value for 0.0033 days or equal to 5 minutes.

4) GTGH - 16 Port GPON Line Card has optimum downtime value for 0.0264 days or equal to 38 minutes.

5) EI8E - 8FE Electrical Interface Card has optimum downtime value for 0.0056 days or equal to 8 minutes.

6) ETC8B - 8E1/T1 CES Card has optimum downtime value for 0.0033 days or equal to 5 minutes.

Several recommendations can be made for the maintenance team, and could become topics for further research:

A. The data input and archiving of failure and/or maintenance of GPON devices should be improved, to increase the accuracy of the calculations.

B. The replacement of GPON devices should be done around the optimum replacement time to minimize the downtime value.

C. For the devices with similar optimum replacement time, preventive maintenance can be done at the same time to minimize cost associated with equipment shut down. Table 9 shows the alternative scheduling of GPON maintenance.

\section{REFERENCES}

D. A. Garvin, "Competing on the Eight Dimensions of Quality," Harvard Business Review, 1987. [Online]. Available: https://hbr.org/1987/11/competing-on-the-eight-dimensions-ofquality. [Accessed: 19-Jun-2019].

[2] International Telecommunication Union, "Gigabit-capable passive optical networks (GPON): General characteristics," 2008.

[3] N. R. Tague, The Quality Toolbox, 2nd Editio. Milwaukee, Wisconsin: American Societyfor Quality, 2005.

[4] L. G. Otaya, "Distribusi Probabilitas Weibull Dan Aplikasinya," TADBIR J. Manaj. Pendidik. Islam, vol. 4, no. 2, pp. 44-66, 2016. R. P. Mcewen and B. R. Parresol, "Moment expressions and summary statistics for the complete and truncated weibull distribution," vol. 0926, 1991.

[6] M. I. Mustajib, U. Nuha, and N. Ansori, "Kebijakan Optimal Penggantian Komponen Berbasis Umur Untuk Preventive Maintenance Pada CNC Plate," no. November, pp. 1-7, 2013.

[7] A. K. . Jardine and A. H. . Tsang, Maintenance, Replacement, and Reliability, 2nd Editio. Boca Raton: CRC Press, 2005. 